\title{
High-Throughput Quality Control Assay for the Solid-Phase Synthe- sis of DNA-Encoded Libraries of Macrocycles
}

\author{
Animesh Roy ${ }^{1}$ and Thomas Kodadek ${ }^{1,2 *}$ \\ ${ }^{1}$ Deluge Biotechnologies, 6671 W. Indiantown Rd., Suite 50-325, Jupiter, FL 33458. \\ ${ }^{2}$ The Scripps Research Institute, Department of Chemistry, 130 Scripps Way, Jupiter, FL 33458.
}

KEYWORDS: DNA-encoded library; macrocycle

\begin{abstract}
There is considerable interest in the development of libraries of scaffold-diverse macrocycles as a source of ligands for difficult targets, such as protein-protein interaction surfaces. A classic problem in the synthesis of high-quality macrocyclic libraries is that some linear precursors will cyclize efficiently while some will not, depending on their conformational preferences. We report here a powerful quality control method that can be employed to readily distinguish between scaffolds that do and do not cyclize efficiently during solid-phase synthesis of thioether macrocycles without the need for tedious liquid chromatography/mass spectrometry analysis. We demonstrate that this assay can be employed to identify largely linear "impurities" in a DNA-encoded library of macrocycles. We also use the method to establish a useful quality control protocol for re-synthesis of putative macrocyclic screening hits.
\end{abstract}

\section{INTRODUCTION}

Libraries of macrocycles are of interest as a potential source of high affinity ligands for difficult to target proteins ${ }^{1-4}$ Indeed, powerful methods for the creation of huge peptide libraries such as ribosome display and phage display have been adapted to make macrocyclic peptides, ${ }^{5-7}$ which have proven to be a rich source of protein ligands. However, most (though not all $^{8,9}$ ) macrocyclic peptides are not cell permeable, which limits their applicability to extracellular targets. Therefore, there has been considerable interest in the development of synthetic libraries of non-peptidic macrocycles with the appropriate physicochemical properties to passively cross membranes. ${ }^{10-12}$ DNA-encoded libraries (DELs) of non-peptidic macrocycles ${ }^{13}$ are of particular interest, since this technology allows the synthesis of libraries as large or larger than those created by phage display. This area was pioneered by Liu and co-workers. They employed DNA-templated chemistry ${ }^{14}$ and a ring-closing Wittig olefination to create libraries of up to 256,000 macrocycles from which ligands for several protein targets have been mined. ${ }^{15,16}$ More recently, Gillingham and co-workers created a library of DNA-encoded macrocycles that involved seven sequential steps, including macrocyclization through amide bond formation.

An important feature of both of these libraries is that they are scaffold-diverse, which is likely to be important in order to provide a general source of ligands for a variety of different protein targets. ${ }^{17}$ However, scaffold diversity brings along with it the problem of disparate efficiencies of macrocyclization of the various molecules in the library, since efficient ring closure depends on the linear precursor accessing a conformation that brings the two end into close proximity. The same issue has also been encountered with some long peptides that adopt a fold that hinders the close approach of the $\mathrm{N}$ - and $\mathrm{C}$-termini. ${ }^{18}$ While variable yields are tolerable in parallel synthesis strategies where reaction products can be purified $^{19-21}$, this is not the case for libraries created by split and pool chemistry, ${ }^{22,23}$ which produces in an intractable mixture of molecules. Indeed, to the best of our knowledge, it is not known what percentage of the molecules in existing macrocyclic DELs are linear "impurities".

We recently developed methodology to create DELs of scaffold-diverse macrocyclic PICCOs ${ }^{24}$ (peptoid-inspired conformationally constrained oligomers) by solid-phase split and pool synthesis. ${ }^{25}$ PICCOs are made using peptoid-like chemistry $^{26}$ in which amines are stitched together with carboxylic acid-containing building blocks that also have a good leaving group elsewhere in the molecule (Scheme 1). Because the carboxylate building blocks employed in the library synthesis have structural features that provide considerable conformational constraint, we anticipated that the aforementioned issue of variable cyclization yields would be significant in the construction of macrocyclic PICCO libraries.

To address this question, we report here a simple but powerful assay for assessment of the efficiency of on-resin cyclization capable of monitoring this process on millions of individual beads. The strategy is shown schematically in Scheme 1 for macrocycles closed via a thioether bond formation. We show here that this method is useful for 
monitoring the progression of an on-resin cyclization reaction at the level of a single compound or a library without the requirement for tedious analysis of reaction products by liquid chromatography/mass spectrometry (LC/MS) after release from the bead.

Scheme 1. Schematic representation of the fluorescence-based assay for monitoring the efficiency on on-resin macrocyclization via thioether formation.
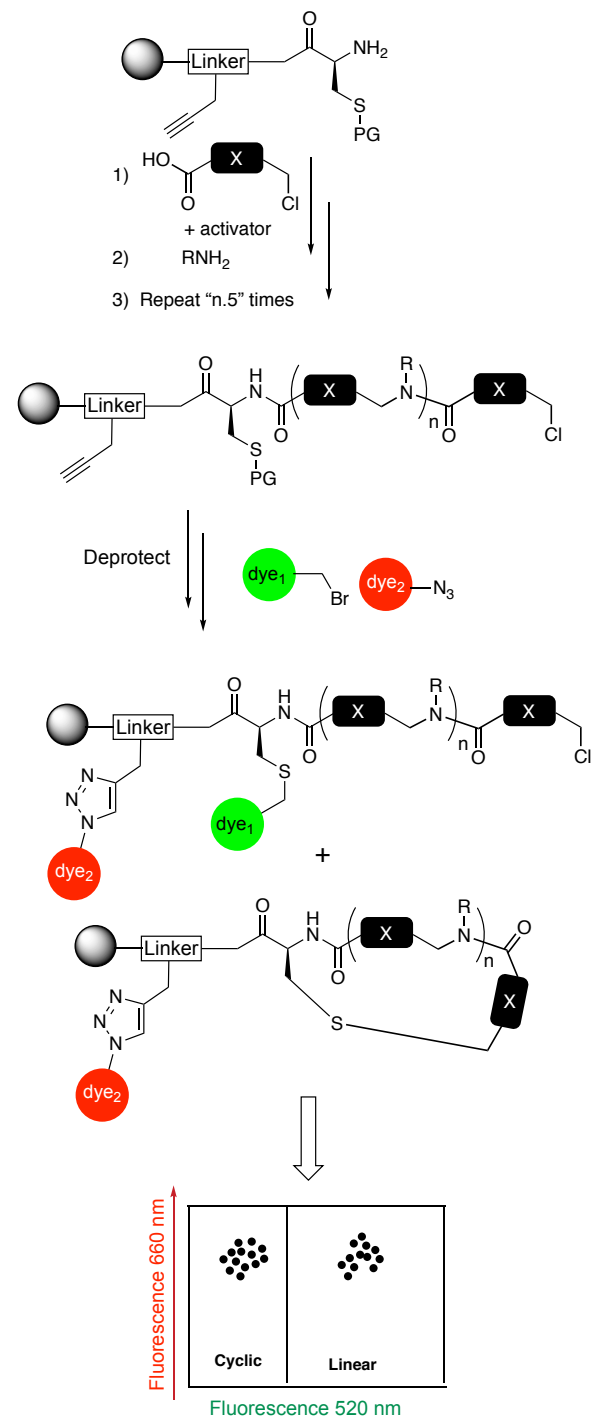

\section{RESULTS \& DISCUSSION}

Establishment of an assay for measuring the ratio of linear/cyclic compounds on-resin. We have created one bead one compound (OBOC) DELs of macrocyclic thioethers (Koesema, et al., submitted). The cyclization reaction involves deprotection of the invariant cysteine followed by incubation in the presence of a mild base, resulting in nucleophilic displacement of the chloride by the thiol (Scheme 1). After staining with a thiol-reactive green dye, beads displaying mostly macrocyclic molecules would have little green color, while those with significant amounts of uncyclized material would fluoresce brightly at this wavelength. To account for the fact that not all beads display the same amount of compound, we also envisioned attaching a red dye to a conserved alkyne in the linker as a normalization (Scheme 1). Since the libraries are constructed on $10 \mu \mathrm{m}$ TentaGel beads, which are about the size of a red blood cell, we imagined that passage of the beads through a flow cytometer would provide a high-throughput method for the determination of the fraction of linear molecules on large numbers of beads.
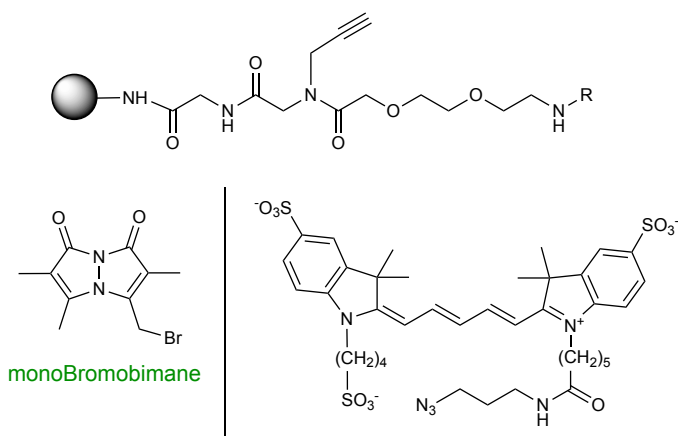

Azido-Cy5-Sulfonate

FACS<smiles>[R][Y][14C](C)=O</smiles>

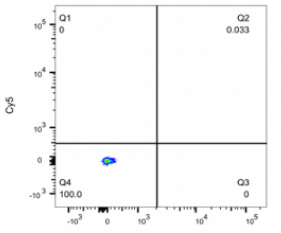<smiles>[R7]C[C@H](NC(=O)CN(CCCOC)C(=O)CN(CCCOC)C(=O)CN(CCCOC)C(C)=O)C(C)=O</smiles>

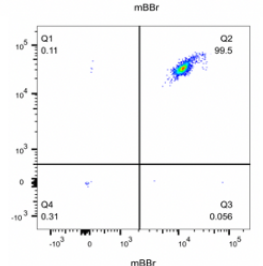<smiles>[R3]CCC(NC(=O)CN(CCCOC)C(=O)CN(CCCOC)C(=O)CN(CCCOC)C(C)=O)C(C)=O</smiles>

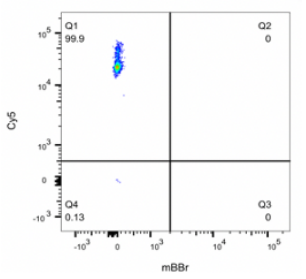

Figure 1. Structures of control compounds and FACS analysis of beads displaying these molecules stained with the dyes shown.

To establish appropriate conditions for this assay, the control compounds shown in Figure 1 were synthesized on 10 $\mu \mathrm{m}$ TentaGel beads. All contain an alkyne unit in the linker. Compound $\mathbf{1}$ has no sulfur atom, while compound $\mathbf{2}$ contains a cysteine and compound $\mathbf{3}$ contains a methionine. $\mathbf{2}$ and $\mathbf{3}$ serve as models for uncyclized and cyclized library members, respectively. We experimented with a variety of thiol-reactive and azide-containing dyes and found that most of them produced an unacceptable level of background staining, even on beads that displayed compound 1 and in the absence of a copper catalyst for Click chemistry. We eventually found that this 
undesirable absorption of dye by the TentaGel beads could be circumvented by using either a "turn on" fluorescent probe or highly hydrophilic dyes, which are far less prone to be trapped by the TentaGel resin. For thiol labeling, we turned to monoBromobimane (mBBr) (Figure 1), a green dye whose fluorescence increases dramatically when the bromide is displaced by a thiol. For conjugation to the conserved alkyne, allowing normalization of the density of reactive sites on the bead, we settled on azido-Cy5-(SO3)3-2 (Figure 1). Different dye concentrations and reaction times were assessed to determine empirically the appropriate amount of staining to be in the linear range of the FACS instrument (SI, Section 2D). Under these conditions, beads displaying compounds $\mathbf{1}$ or $\mathbf{3}$ acquired little or no green fluorescence when treated with $\mathrm{mBBr}$, whereas most of the beads displaying cysteine-containing compound 2 became intensely fluorescent (Figure 1B). Beads subjected to the Click reaction with azido-Cy5-(SO3)3-2 fluoresced intensely in the red channel. Only a modest level of variability in the degree of staining of the $10 \mu \mathrm{m}$ TentaGel beads with azido-Cy5-(SO3)3-2 was observed, presumably reflecting a relatively homogenous population of beads with respect to the number of reactive sites. This is in contrast to larger TentaGel beads, in which this value can vary by over 20 -fold from bead to bead. ${ }^{27}$ Therefore, some of the experiments below employed this normalization dye, while some did not.

Monitoring macrocyclization on-resin. With these conditions in hand, we asked if this method was suitable for following the progress of a macrocyclization reaction of a single compound on resin. Compound DDA (Figure 2A), was subjected to standard macrocyclization conditions. Specifically, the thiol was exposed by treatment with DTT $(100 \mathrm{mM})$ and $\mathrm{N}$-methyl morpholine $(100 \mathrm{mM})$ in DMF for five minutes, three times. After buffer exchange (SI, Section 2B) the beads were then incubated in a buffered aqueous solution. At various times afterward, an aliquot of $10 \mu \mathrm{m}$ beads was removed from the reaction and stained with $\mathrm{mBBr}$ for an hour. Following a wash, the fluorescence intensity of these beads was analyzed using a flow cytometer. As shown in Figure 2B, panel B4, the green fluorescence intensity on beads treated with $\mathrm{mBBr}$ dye two hours after exposure of the thiol was reduced greatly in comparison to beads displaying the linear control molecule 2 (Figure 2B, panel B2), indicating a fast cyclization rate. Indeed, for this molecule the reaction appeared almost complete in two hours. With additional time, the level of staining decreased slowly and by 16 hours following thiol exposure, about $99 \%$ of the beads were 50 -fold less fluorescent than the control linear model (panels B4-B6).

LC/MS analysis of the reaction generally corroborates this interpretation of the flow data. Scaffold DDA was synthesized on larger $160 \mu \mathrm{m}$ TentaGel RAM beads with a linker that facilitates ionization in the mass spectrometer. At various times following exposure of the thiol, an aliquot of the beads was treated with excess benzyl bromide for two hours to "freeze" the cyclization reaction prior to release of the compounds from the beads $(90 \%$ TFA, $2.5 \%$ TIPS, $2.5 \%$ thioanisole, $5.0 \% \mathrm{DCM})$. The ratio of linear and macrocyclic compounds was then assessed by LC/MS. Using this assay, the reaction was $24 \%$ complete after two hours, $48 \%$ complete after eight hours and reached $81 \%$ conversion after sixteen hours. This is slower than the same reaction on the smaller TentaGel resin, but this is perhaps not surprising, since it is impossible to conduct identical experiments on the two resins. The compounds are attached to the bead by different linkers and there is likely a significant difference in the fraction of sites buried in the polystyrene core versus the hydrophilic PEGylated coating on the surface of the beads.

To investigate if we are losing signal due to thiol oxidation rather than macrocyclization, acetylated scaffold DD-STMP was incubated at $37^{\circ} \mathrm{C}$ for 16 hours in PBST buffer (Figure 2D) after thiol deprotection. Staining with $\mathrm{mBBr}$ dye produced almost identical fluorescence intensity as linear standard (Panel B10 vs B2). This demonstrates thiol oxidation does not occur to a substantial degree in the time frame of the experiment. Similarly, benzylated product was observed in mass analysis from $160 \mu \mathrm{m}$ beads after treatment with benzyl bromide (SI, Section 4C). We conclude that the dye staining/flow cytometry assay is suitable for monitoring the progress of a macrocyclization reaction.

Macrocyclization efficiency of different scaffolds. A set of 64 molecules with the general structure shown in Figure 3A were prepared by parallel solid-phase synthesis on both $10 \&$ $160 \mu \mathrm{m}$ TentaGel resin in a microtiter plate format. AzidoCy5- $\left(\mathrm{SO}_{3}\right)_{2}$ was added to the conserved alkyne in the linker for $10 \mu \mathrm{m}$ beads prior to thiol deprotection. The $64 \mathrm{com}-$ pounds represent different scaffolds, each with a distinct conformational preference. Each compound was subjected to the cyclization reaction conditions for eight hours, followed by staining the $10 \mu \mathrm{m}$ beads with $\mathrm{mBBr}$ to assess the degree of cyclization on-resin. At the same time, the analogous $160 \mu \mathrm{m}$ beads were treated identically for eight hours, the compounds cleaved from resin and processed for LC/MS analysis (SI, Section 9). Fig. 3B shows a summary of the results from the fluorescence analysis (for all of the primary data see Supplementary Section $5 \&$ 9). Most of the compounds in this small library cyclized efficiently, with at least $95 \%$ of the beads being located in a quadrant of the flow plot indicating a low level of staining with $\mathrm{mBBr}$ and thus a small amount of linear starting material. The primary data for four of these compounds is shown in Figure 3D. In contrast, a few of the compounds (highlighted in yellow in Fig. 3B) provided a different result. Eight hours after thiol exposure, the level of $\mathrm{mBBr}$ staining suggested that the beads displayed a substantial amount of linear material, but less than that displayed by the linear control. The most reasonable interpretation of these data is that these compounds cyclize more slowly, and only partial conversion is realized after eight hours. Indeed, when beads displaying four of these compounds were stained 16 hours after thiol exposure, the level of staining had decreased to the level expected of a high yield macrocyclization reaction (Fig. 3E). Again, the LC/MS data from the analogous reactions on the $160 \mu \mathrm{m}$ TentaGel RAM beads largely corroborate the interpretation of the flow data, with some variability in rate. Eight hours after thiol exposure, the beads were released from the resin by treatment with TFA. The majority of the scaffolds $(50 / 64)$ appeared to have completed cyclization ( $>90 \%)$ while others progress more slowly (SI, Table T5).

In general, the scaffolds that cyclize more slowly have acid $\mathrm{B}$ (Fig. 3A) at the terminus. In these reactions the thiol must attack a secondary carbon rather than a primary benzylic or allylic center, presumably explaining the slower rate of ring closure. 
A.
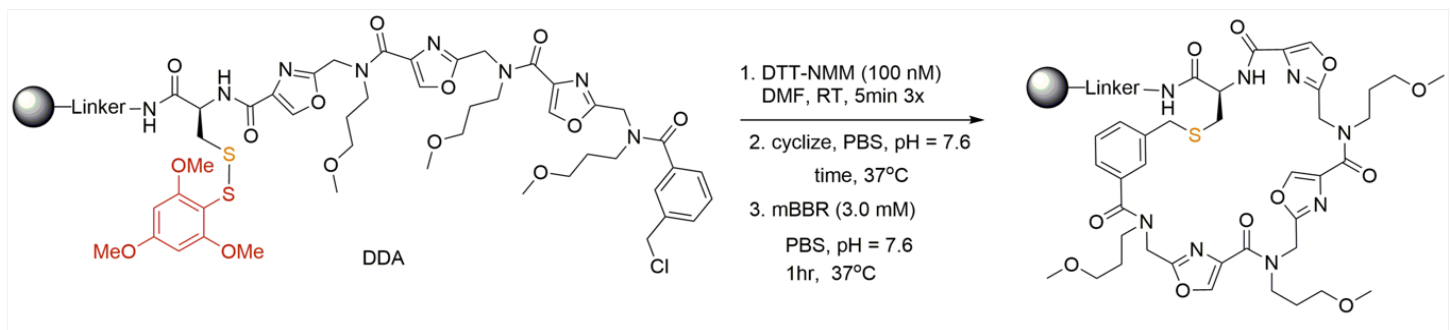

cyclo-DDA
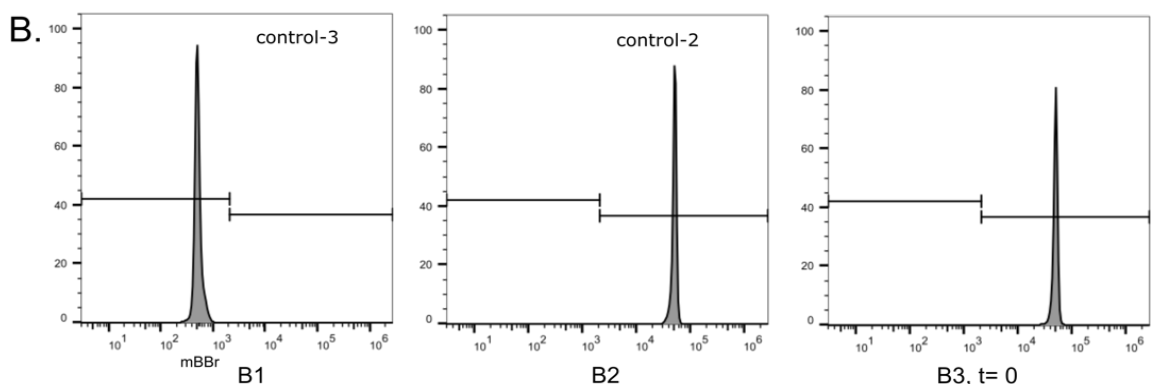

C.

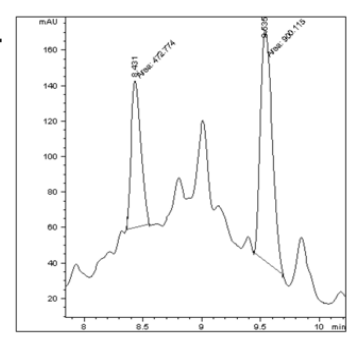

C1, t $=2 \mathrm{hrs}$
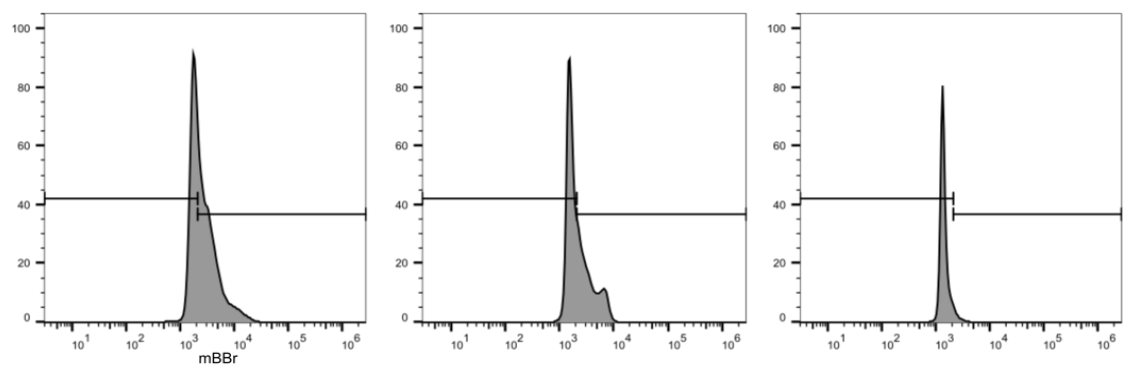

B4, $\mathrm{t}=2 \mathrm{hrs}$

B5, t= 4hrs
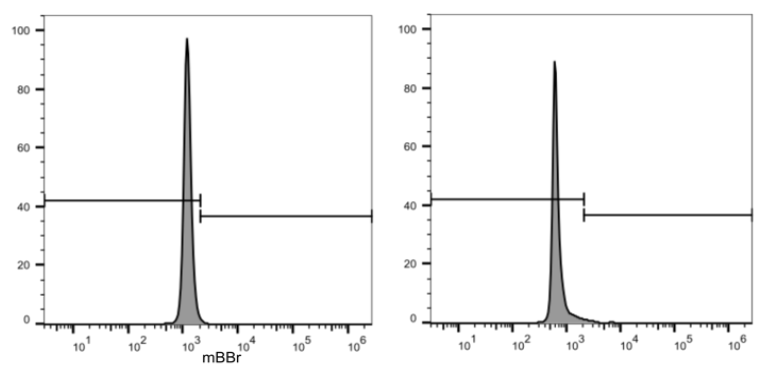

$B 6, t=6 h r s$

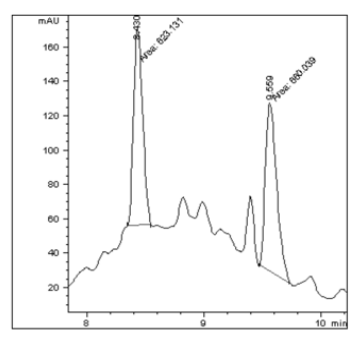

$\mathrm{C} 2, \mathrm{t}=8 \mathrm{hrs}$

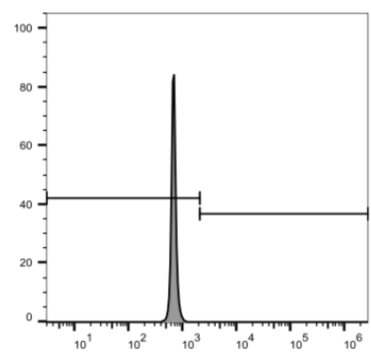

$\mathrm{B} 8, \mathrm{t}=10 \mathrm{hrs}$

B9, $\mathrm{t}=16 \mathrm{hrs}$

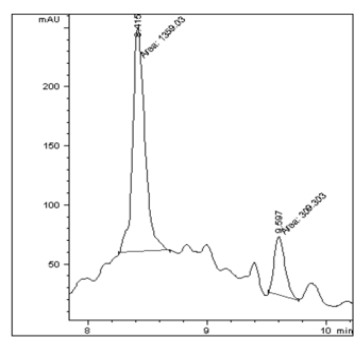

C3, $\mathrm{t}=16 \mathrm{hrs}$
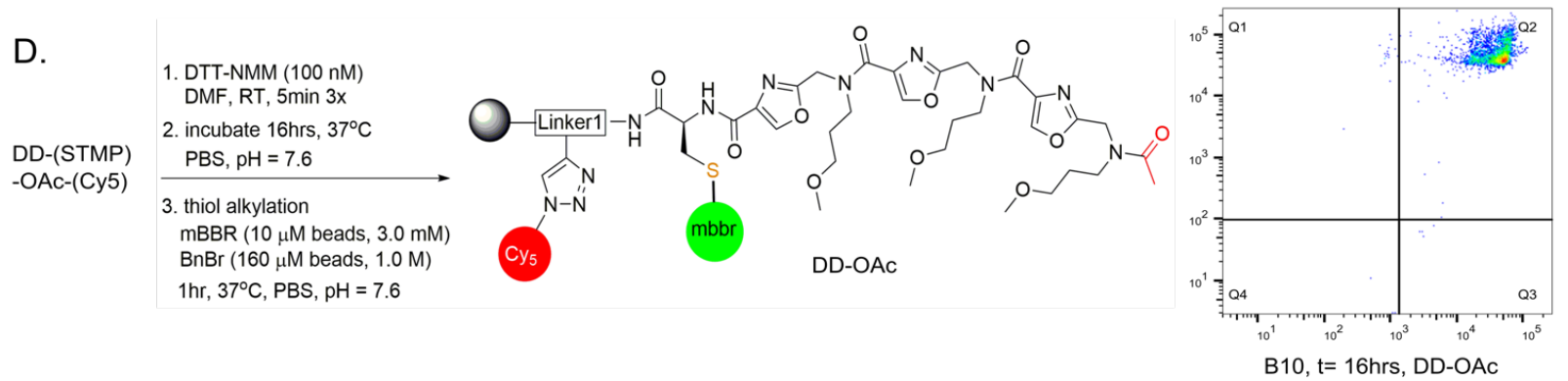

Figure 2: Monitoring the progress of macrocylization of DDA. (A) Reaction conditions used for the experiment. (B) Flow cytometer histograms showing the level of staining of $10 \mu \mathrm{m}$ TentaGel beads with $\mathrm{mBBr}$ at the times indicated (post-deprotection). Panel B1 shows beads displaying 3 (the methionine control) and Panel B2 shows beads displaying 2 (the cysteine control; see Fig. 1). Panels B3-10 show $\mathrm{mBBr}$ staining of beads displaying DDA. (C) Progress of DDA cyclization on $160 \mu \mathrm{m}$ TentaGel ebads as monitored by LC-mS analysis after alkylation of free thiol with benzylbromide and release of the compound from the bead. (D) mBBr staining and FACS analysis of 10 $\mu \mathrm{m}$ TentaGel beads displaying DD-OAc, a molecule that cannot cyclize. Even 16 hours post-deprotection, the thiol is robustly alkylated, by $\mathrm{mBBr}$, showing that oxidation of the sulfur over this time period is not significant. 
A.

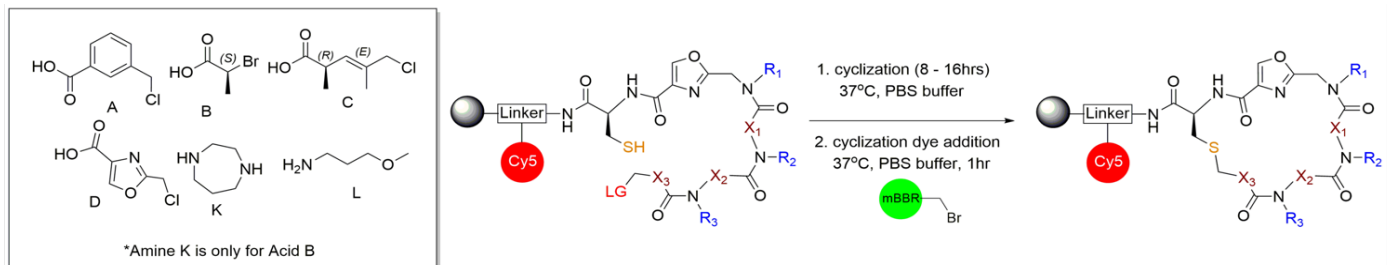

\begin{tabular}{|c|c|c|c|c|c|c|c|}
\hline AAA & ABA & ACA & ADA & $A A B$ & ABB & $\mathrm{ACB}$ & ADB \\
\hline BAA & BBA & BCA & BDA & $\mathrm{BAB}$ & BBB & BCB & BDB \\
\hline CAA & CBA & CCA & $\mathrm{CDA}$ & $C A B$ & CBB & CCB & $\mathrm{CDB}$ \\
\hline DAA & DBA & DCA & DDA & DAB & DBB & DCB & DDB \\
\hline AAD & ABD & $A C D$ & ADD & AAC & $A B C$ & ACC & $A D C$ \\
\hline BAD & BBD & $\mathrm{BCD}$ & BDD & BAC & BBC & BCC & BDC \\
\hline CAD & CBD & $C C D$ & $\mathrm{CDD}$ & CAC & $\mathrm{CBC}$ & CCC & $\mathrm{CDC}$ \\
\hline DAD & DBD & DCD & DDD & DAC & DBC & DCC & DDC \\
\hline
\end{tabular}
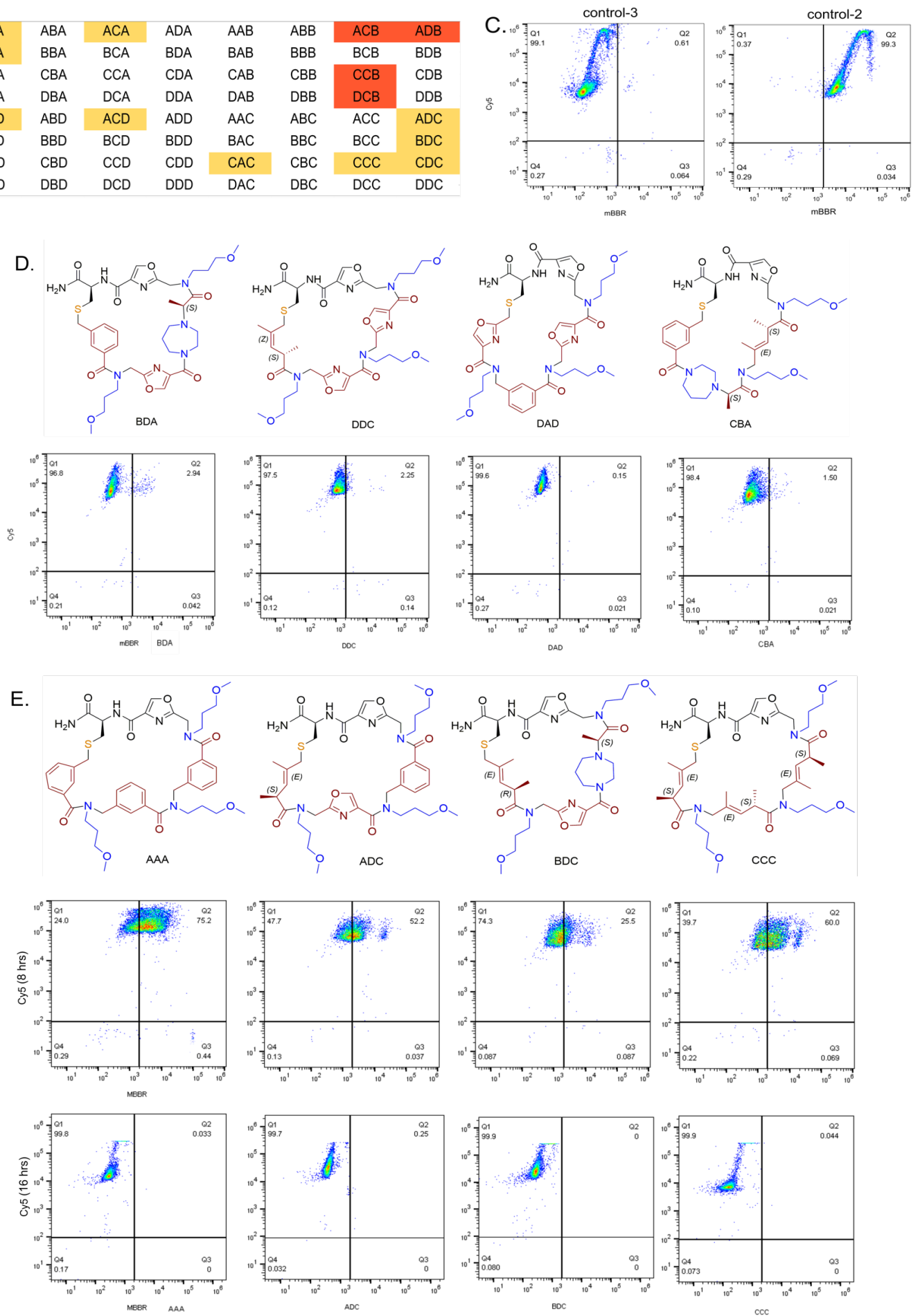

Figure 3: Analysis of the efficiency of macrocyclization of 64 PICCO scaffolds created by parallel solid-phase synthesis. (A) Structures of the building blocks employed (box) and the protocol employed for macrocylization. Diamine K was always employed following acid B, while amine $\mathrm{L}$ was always used following all of the other acids. This is because acylation of $\mathrm{N}$-alkylated alanines is difficult. ${ }^{28} \mathrm{Cy} 5$-azide dye was attached to alkyne handle of the linker, the thiol protection was removed and the beads were stained with $\mathrm{mBBr}$ eight hours later. 
Bead fluorescence was then analyzed using a flow cytometer. (B) Summary of ring-closure status of 64 scaffolds from FACS \& LCMS analysis. Cells in yellow indicate scaffolds that had between 5-40\% linear material after 8 hours while red indicates scaffolds that had more than $40 \%$ linear material present. The rest of the scaffolds did not show any detectable linear material. (C) FACS plots for the control molecules 2 and 3 (see Fig. 1). (D) FACS plots for selected scaffolds that showed a high degree of cyclization after eight hours. (E) Selected scaffolds that showed incomplete cyclization after eight hours (top row of FACS plots) but completed macrocyclization by 16 hours (bottom row of FACS plots).

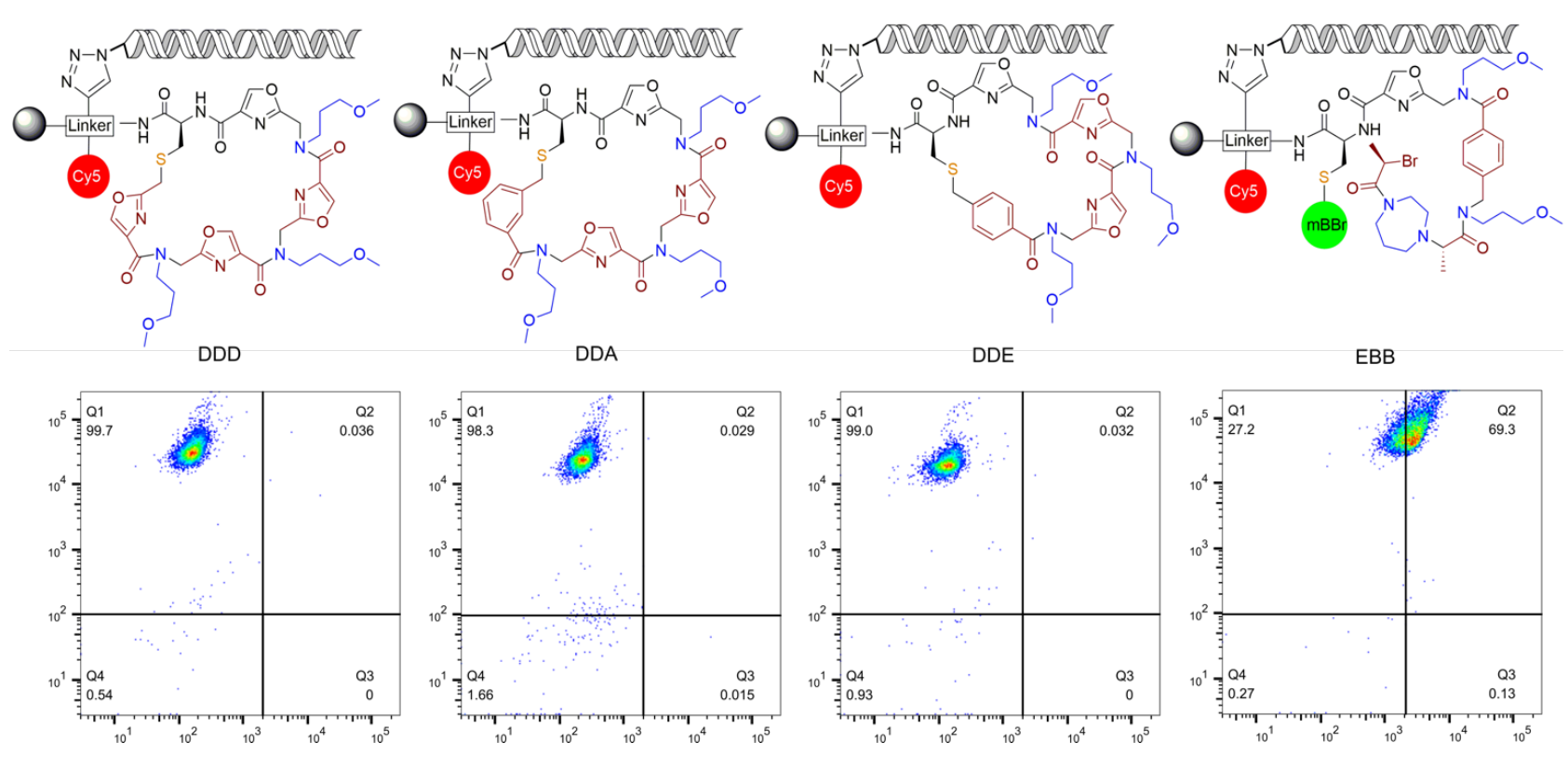

Figure 4: Staining in the presence of DNA encoding tags to monitor on-resin cyclization. The FACS blots show the level of staining of beads eight hours after removal of the thiol protecting group.

Effect of DNA encoding tags. We next turned to applying this method to monitor the on-resin macrocyclization of DNA-encoded compounds. In this platform, less than $1 \%$ of the molecules on the bead have a DNA encoding tag appended to the invariant linker. Nonetheless, we have found that some reactions are affected by the presence of the DNA.

Azido-headpiece DNA was clicked to a modified version of compound-1 (SI, Figure S10) and a full length test-DNA encoding tag was ligated. After three acylations and aminations the linear precursor was subjected to $\mathrm{CuAAC}$ reaction to link Cy5-azide dye. Encoding DNA tags from these beads could not be amplified (data not shown) indicating that a late stage $\mathrm{CuAAC}$ reaction is not suitable for this assay. Therefore, we turned to a strain-promoted Click reaction. For this, 1,3-diazidopropane was clicked to the invariant alkyne during azidoHDNA addition via CuAAC. A full-length test-DNA tag was ligated onto the headpiece and chemistry was carried out to construct the linear precursor to cyclization. Resin was suspended in $\mathrm{CRB}$ buffer, and a cyclooctene-containing dye (Cy5-DBCO; $0.11 \mathrm{mM}$, DMSO) was added and incubated at $37^{\circ} \mathrm{C}, 16$ hours to label the azide in the linker. We found that the conditions for $\mathrm{mBBr}$ labeling described above for beads lacking DNA resulted in a significantly lower labeling efficiency for encoded beads. Reoptimization showed that a higher concentration of dye $(9 \mathrm{mM})$ and a longer reaction time (two hours) provided a level of labeling commensurate to what had been observed with the beads lacking encoding tags and to retain sufficient DNA tags for PCR amplification.

With these conditions set, we assessed the utility of the fluorescent method to monitor macrocyclic thioether formation on beads with encoding tags. Four compounds (DDD, DDA, $\mathrm{DDE}$ and $\mathrm{EBB}$ ) were constructed on both 10 and $160 \mu \mathrm{m}$ TentaGel beads with DNA tags and subjected to the standard cyclization conditions (SI, Section 6). The degree of cyclization was determined using the flow assay $(10 \mu \mathrm{m})$ and by mass analysis after release from the beads $(160 \mu \mathrm{m})$. In the latter case, any free thiol was first quenched by the addition of excess benzyl bromide $\left(1.0 \mathrm{M}\right.$ for $1 \mathrm{hr}$ at $\left.37^{\circ} \mathrm{C}\right)$ prior to TFAmediated release of the compound from the resin to prevent cyclization during the processing and analysis period.

Two of these molecules, DDD and DDA, were shown to be excellent cyclization substrates in the absence of the DNA tag (Figure 3B), and we anticipated that compound DDE would also likely cyclize well given the presence of the highly reactive bromomethyl benzene at the chain terminus. In contrast, we hypothesized that compound EBB might cyclize more slowly given the presence of a secondary bromide at the terminus. As shown in Figure 4, flow analysis suggested that compounds DDD, DDA and DDE cyclized in almost 
quantitative yield in 16 hours, whereas the production of the thioether from EBB was still in progress, as anticipated. This interpretation was corroborated via mass analysis of the material synthesized on the $160 \mu \mathrm{m}$ beads (SI, Section 6A). We conclude that this assay is suitable for tracking the cyclization of DNA-encoded compounds on-resin.
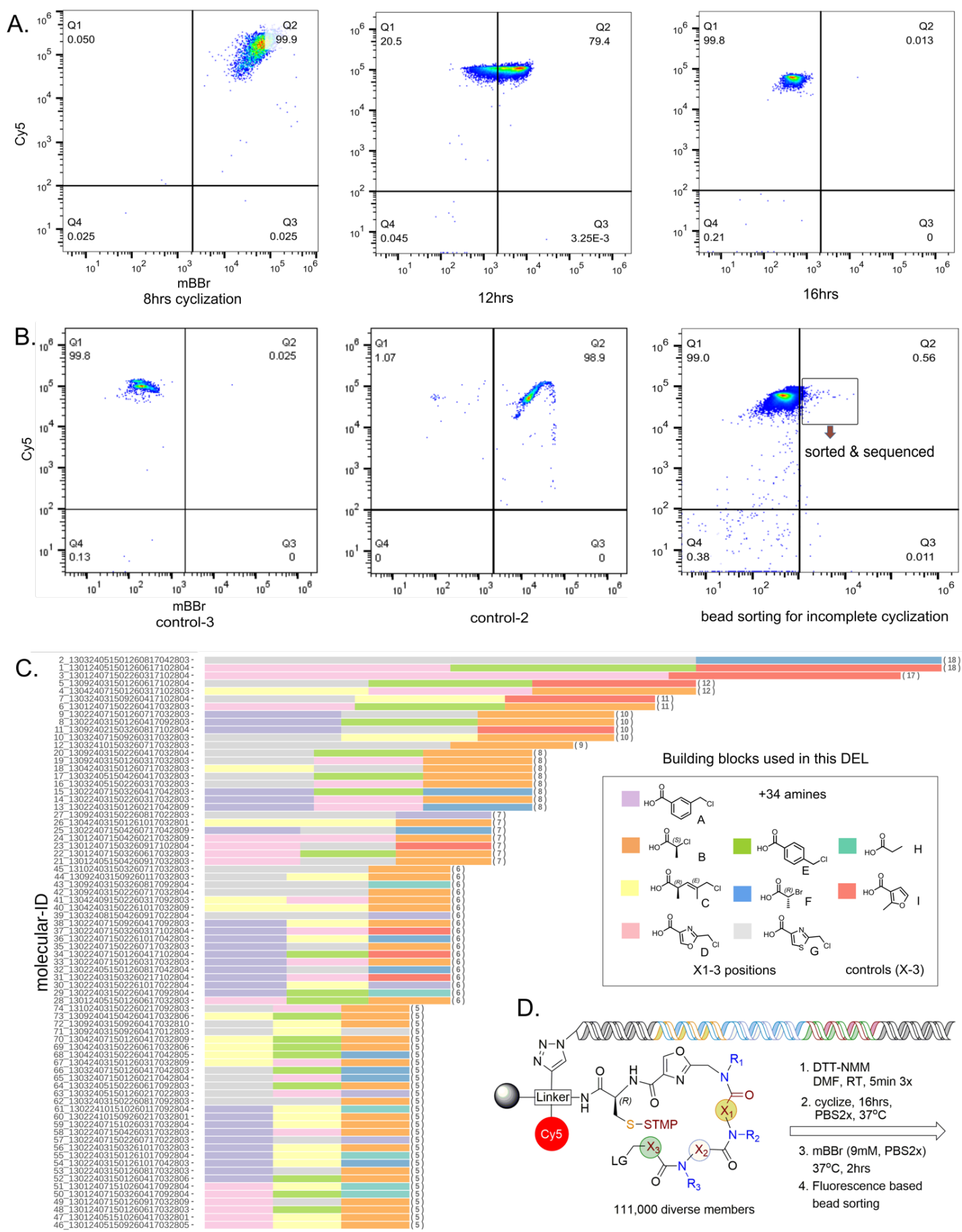

D.

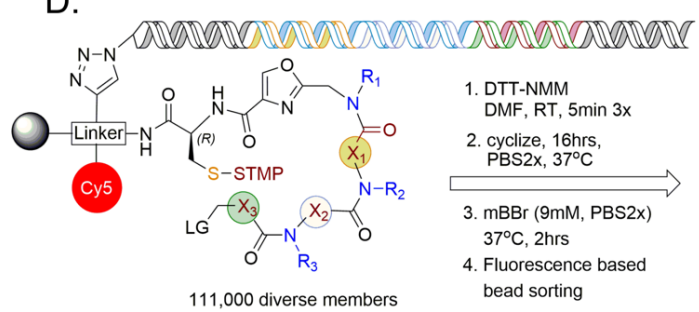

Figure 5: High-throughput flow-cytometry based quality control assay for ring closure of DNA encoded OBOC macrocyclic library. After linear precursor synthesis, a portion of the DEL was separately stained with Cy 5 dye, cyclized and stained again with $\mathrm{mBBr}$ dye for analysis. (A) On-resin cyclization progress was monitored to ensure majority of the library member cyclized. (B) Beads that have incomplete cyclization were separated. (C) After deep sequencing, difficult to cyclize scaffolds were arranged by count (up to 5 copies, 74 scaffolds). Each line indicates a combination of three acids at position X1-3 for a scaffold along with its molecular ID (left) and the number of times it was present in the collected pool (right). (D) Acids A - G and amines were used in position X1-X3 to construct DEL scaffolds. 4\% of DEL members were capped with acid H \& I to keep them as linear controls for FACS analysis. 
Quality control of a DEL of macrocycles. Finally, we proceeded to employ the fluorescent labeling/flow cytometry assay to analysis of macrocyclization in the context of a DEL synthesis. As shown in Figure 5, seven carboxylic acids and 34 amines (SI, Section 7) were employed to create the DEL by split and pool synthesis on $10 \mu \mathrm{m}$ TentaGel beads. Library synthesis began with $25 \mathrm{mg}$ of $10 \mu \mathrm{m}$ TentaGel beads by attaching Azido-headpiece $\mathrm{DNA}^{29,30}(<1 \%)$ and 1,3-diazidopropane $(>50 \%)$ at once via a copper-catalyzed reaction to the alkyne part of the linker. After 3 cycles of split \& pool chemistry and consecutive enzymatic ligations of encoding DNA tags, 110,000 diverse linear precursor were prepared. About $4 \%$ of the library members were capped with acid $\mathrm{H}$, and I (Figure 5C \& D) at position 3. Since these groups lack a leaving group, they cannot cyclize and thus function as internal linear control molecules in the library.

The thiol protecting STMP group was removed and the beads were incubated at $37^{\circ} \mathrm{C}$ for ring closure. A portion of the library (about $1 / 5$ th) was reacted separately with Cy5$\mathrm{DBCO}$ dye prior to thiol deprotection and monitored for ring closure over time. Aliquots $(\sim 10,000$ beads) were removed 8 , 12 and 16 hours later, stained with $\mathrm{mBBr}$, and then analyzed using a flow cytometer. As shown in Figure 5A, almost all of the beads had substantial amounts of thiol remaining after 8 hours. Four hours later, substantial ring closure was reflected by the lower average level of green fluorescence. By 16 hours post-deprotection, almost all of the beads evinced a low level of fluorescence in the green channel, indicating that macrocyclization was complete for the vast majority of compounds in the library. A fluorescence based gate was established to collect beads $(\sim 6500)$ that had higher fluorescence intensity than DNA-linked control 3 (Figure 5B). The DNA encoding tags on these beads were deep sequenced to identify acid building blocks contained in these difficult-to-cyclize scaffolds. Fig. $5 \mathrm{C}$ represents these data graphically in order of the number of times that combination of main chain building blocks was found in the difficult-to-cyclize pool (Figure 5C). Linear control compounds with acid I at the last position X3 (Figure 5D) were recovered with the highest count (18 copies) and molecules with acid $\mathrm{H}$ at position $\mathrm{X} 3$ were also found, as expected.

2-chloro- or 2-bromo-propionic acid were, by far, the most commonly found residues at position X3 in the molecules that were capable of cyclizing but did so slowly. Several combinations of different acids at X1 and X2 were seen linked to the halopropionic acids in this pool, including scaffolds that had higher levels of incomplete cyclization in previous the LCMS analysis (ACB, ADB, CCB, DCB (Fig. 3B)) (Fig. 5C). This observation indicates again that halide displacement on a secondary center is simply slower than from a primary center. Having said this, certain combination of acids in position $\mathrm{X} 1$ and $\mathrm{X} 2$ in combination with halopropionic acids at position $\mathrm{X} 3$ appear to hinder cyclization more than others. For example, $\mathrm{X} 1=\mathrm{X} 2=\mathrm{G}, \mathrm{X} 3=\mathrm{B}$ or $\mathrm{F}$ is extremely common (Fig. 5C).

The scaffolds enriched in the difficult-to-cyclize pool had a number of different amine-derived side chains, suggesting that the main chain carboxylic acid-derived building blocks are the dominant players in determining cyclization efficiency, as one might have expected.

What is perhaps most striking about these data, however, is what is not found commonly in the difficult-to-cyclize pool, and that is any scaffold with a unit other than chloro- or bromopropionic acids or the uncyclizable control acids at position X3. There are only a few exceptions. This shows clearly that most of the diverse scaffolds in the library cyclize quite efficiently, showing that high-quality, yet scaffold-diverse, macrocyclic PICCO libraries can be constructed.

\section{CONCLUSION}

In summary we have developed a flow cytometer-based assay to analyze the on-resin ring-closure process for OBOC DNA-encoded thioether macrocycles. We have demonstrated this powerful methodology is able to monitor the chemistry that occurs on hundreds of thousands or even millions of individual $10 \mu \mathrm{m}$ TentaGel beads. This makes it possible to quality control even very large DELs of macrocycles, something that is not currently possible using standard techniques. While this study focused on monitoring the efficiency of thioether formation by staining unreacted sulfhydryls with a thiol-reactive dye, it should be possible to adapt this approach to almost any ring-closing chemistry so long as one of the reacting partners is unique in the molecule. It also seems reasonable to suggest that this scheme could be adapted to identify difficult-to-cyclize molecules in DELs produced by solution-phase split and pool synthesis, where each molecule is affixed to a DNA tag and the compounds are part of an intractable mixture. ${ }^{29}$ In this case, the entire library could be treated, for example, with a thiol-reactive biotinylating agent, for example. The biotinylated molecules could be pulled out of solution with immobilized streptavidin and the corresponding encoding tags deep sequenced to identify linear scaffolds. Therefore, we believe that this basic assay format will be of general utility in the quality assessment of macrocyclic DELs.

\section{ASSOCIATED CONTENT}

\section{Supporting Information}

Methods and supplementary data are provided.

\section{AUTHOR INFORMATION}

\section{Corresponding Author}

* To whom correspondence may be addressed at: Thomas.Kodadek@DelugeBiotechnologies.com

\section{Author Contributions}

The manuscript was written through contributions of all authors. / All authors have given approval to the final version of the manuscript. / \$These authors contributed equally. (match statement to author names with a symbol)

\section{Funding Sources}

This work was supported by a SBIR grant from the NIH (GM130164).

\section{Conflict of Interest Statement}

T.K. is a significant shareholder in Deluge Biotechnologies, which is commercializing DNA-encoded libraries of macrocycles.

\section{ACKNOWLEDGMENT}

We thank Dr. Eric Koesema for technical support with the molecular biology aspects of this work and Dr. Ofelia Utset for administrative support. 
1. Driggers, E. M.; Hale, S. P.; Lee, J.; Terrett, N. K., The exploration of macrocycles for drug discovery--an underexploited structural class. Nat Rev Drug Discov 2008, 7 (7), 608-24.

2. Whitty, A.; Viarengo, L. A.; Zhong, M., Progress towards the broad use of non-peptide synthetic macrocycles in drug discovery.

Organic \& Biomolecular Chemistry 2017, 15 (37), 7729-7735.

3. Bauer, R. A.; Wurst, J. M.; Tan, D. S., Expanding the range of "druggable" targets with natural product-based libraries: an academic perspective. Curr. Op. Chem. Biol. 2010, 14, 308-314.

4. Dandapani, S.; Marcaurelle, L. A., Grand Challenge Commentary: Accessing new chemical space for 'undruggable' targets. Nature

Chemical Biology 2010, 6 (12), 861-863.

5. Reid, P. C.; Goto, Y.; Katoh, T.; Suga, $\mathrm{H}$., Charging of tRNAs using ribozymes and selection of cyclic peptides containing thioethers. Methods Mol Biol 2012, 805, 33548.

6. Vinogradov, A. A.; Yin, Y.; Suga, H., Macrocyclic Peptides as Drug Candidates: Recent Progress and Remaining Challenges. Journal of the American Chemical Society 2019, 141 (10), 4167-4181.

7. Heinis, C.; Rutherford, T.; Freund, S.; Winter, G., Phage-encoded combinatorial chemical libraries based on bicyclic peptides. Nature Chem Biol 2009, 5 (7), 502-7.

8. Bockus, A. T.; McEwan, C. M.; Lokey, R. S., Form and function in cyclic peptide natural products: A pharmacokinetic perspective. Curr. Top. Med. Chem. 2013, 13, 821-836.

9. Fouché, M.; Schäfer, M.; Berghausen, J.; Desrayaud, S.; Blatter, M.; Piéchon, P.;

Dix, I.; Martin Garcia, A.; Roth, H.-J., Design and Development of a Cyclic Decapeptide

Scaffold with Suitable Properties for Bioavailability and Oral Exposure. ChemMedChem 2016, 11 (10), 1048-1059.

10. Villar, E. A.; Beglov, D.; Chennamadhavuni, S.; Porco, J. A.; Kozakov,

\section{REFERENCES}

D.; Vajda, S.; Whitty, A., How proteins bind macrocycles. Nature Chemical Biology 2014, 10 (9), 723-731.

11. Over, B.; Matsson, P.; Tyrchan, C.; Artursson, P.; Doak, B. C.; Foley, M. A.; Hilgendorf, C.; Johnston, S. E.; Lee, M. D.; Lewis, R. J.; McCarren, P.; Muncipinto, G.; Norinder, U.; Perry, M. W. D.; Duvall, J. R.; Kihlberg, J., Structural and conformational determinants of macrocycle cell permeability. Nature Chemical Biology 2016, 12 (12), 10651074.

12. Pye, C. R.; Hewitt, W. M.; Schwochert, J.; Haddad, T. D.; Townsend, C. E.; Etienne, L.; Lao, Y.; Limberakis, C.; Furukawa, A.; Mathiowetz, A. M.; Price, D. A.; Liras, S.; Lokey, R. S., Nonclassical Size Dependence of Permeation Defines Bounds for Passive Adsorption of Large Drug Molecules. Journal of Medicinal Chemistry 2017, 60 (5), 16651672.

13. Connors, W. H.; Hale, S. P.; Terrett, N. K., DNA-encoded chemical libraries of macrocycles. Current Opinion in Chemical Biology 2015, 26, 42-47.

14. Li, X.; Liu, D. R., DNA-templated organic synthesis: Nature's strategy for controlling chemical reactivity applied to synthetic molecules. Angew Chem Int Ed Engl 2004, 43, 4848-4870.

15. Kleiner, R. E.; Dumelin, C. E.; Tiu, G. C.; Sakurai, K.; Liu, D. R., In vitro selection of a DNA-templated small-molecule library reveals a class of macrocyclic kinase inhibitors.

J Am Chem Soc 2010, 132 (33), 11779-91.

16. Usanov, D. L.; Chan, A. I.; Maianti, J.

P.; Liu, D. R., Second-generation DNA-

templated macrocycle libraries for the discovery of bioactive small molecules. Nat Chem 2018, 10 (7), 704-714.

17. Dickson, P.; Kodadek, T., Chemical composition of DNA-encoded libraries, past present and future. Org Biomol Chem 2019, 17 (19), 4676-4688.

18. Jagasia, R.; Holub, J. M.; Bollinger, M.; Kirshenbaum, K.; Finn, M. G., Peptide Cyclization and Cyclodimerization by CuI- 
Mediated Azide-Alkyne Cycloaddition. The Journal of Organic Chemistry 2009, 74 (8), 2964-2974.

19. Mortensen, K. T.; Osberger, T. J.; King, T. A.; Sore, H. F.; Spring, D. R., Strategies for the Diversity-Oriented Synthesis of Macrocycles. Chemical Reviews 2019, 119 (17), 10288-10317.

20. Nielsen, T. E.; Schreiber, S. L., Towards the optimal screening collection: a synthesis strategy. Angew Chem Int Ed Engl 2008, 47 (1), 48-56.

21. Isidro-Llobet, A.; Hadje Georgiou, K.; Galloway, W. R. J. D.; Giacomini, E.; Hansen, M. R.; Méndez-Abt, G.; Tan, Y.S.; Carro, L.; Sore, H. F.; Spring, D. R., A diversity-oriented synthesis strategy enabling the combinatorialtype variation of macrocyclic peptidomimetic scaffolds. Organic \& Biomolecular Chemistry 2015, 13 (15), 4570-4580.

22. Lam, K. S.; Salmon, S. E.; Hersh, E. M.; Hruby, V. J.; Kazmierski, W. M.; Knapp,

R. J., A new type of synthetic peptide library for identifying ligand-binding activity. Nature

1991, 354, 82-84.

23. Houghten, R. A.; Pinilla, C.; Blondelle, S. E.; Appel, J. R.; Dooley, C. T.; Cuervo, J. $\mathrm{H}$., Generation and use of synthetic peptide combinatorial libraries for basic research and drug discovery. Nature 1991, 354 (6348), 84-6. 24. Kodadek, T.; McEnaney, P. J., Towards vast libraries of scaffold-diverse, conformationally constrained oligomers. Chem Commun (Camb) 2016, 52 (36), 6038-59.

25. Koesema, E.; Roy, A.; Kodadek, T., Synthesis and screening of DNA-encoded libraries of non-peptidic thioether macrocycles. J. Amer. Chem. Soc. 2020, 142, Submitted. 26. Zuckermann, R. N.; Kerr, J. M.; Kent, S. B. H.; Moos, W. H., Efficient method for the preparation of peptoids (oligo(N-substituted glycines)) by sub-monomer solid-phase synthesis. J. Amer. Chem. Soc. 1992, 114, 10646-10647.

27. Doran, T. M.; Gao, Y.; Mendes, K.;

Dean, S.; Simanski, S.; Kodadek, T., The utility of redundant combinatorial libraries in distinguishing high and low quality screening hits. ACS Comb. Sci. 2014, 16, 259-270.

28. Gao, Y.; Kodadek, T., Synthesis and screening of stereochemically diverse combinatorial libraries of peptide tertiary amides. Chem. \& Biol. 2013, 20, 360-369.

29. Clark, M. A.; Acharya, R. A.; AricoMuendel, C. C.; Belyanskaya, S. L.; Benjamin, D. R.; Carlson, N. R.; Centrella, P. A.; Chiu, C. H.; Creaser, S. P.; Cuozzo, J. W.; Davie, C. P.; Ding, Y.; Franklin, G. J.; Franzen, K. D.; Gefter, M. L.; Hale, S. P.; Hansen, N. J.; Israel, D. I.; Jiang, J.; Kavarana, M. J.; Kelley, M. S.; Kollmann, C. S.; Li, F.; Lind, K.; Mataruse, S.; Medeiros, P. F.; Messer, J. A.; Myers, P.; O'Keefe, H.; Oliff, M. C.; Rise, C. E.; Satz, A. L.; Skinner, S. R.; Svendsen, J. L.; Tang, L.; van Vloten, K.; Wagner, R. W.; Yao, G.; Zhao, B.; Morgan, B. A., Design, synthesis and selection of DNA-encoded smallmolecule libraries. Nature Chem Biol 2009, 5 (9), 647-54.

30. MacConnell, A. B.; McEnaney, P. J.;

Cavett, V. J.; Paegel, B. M., DNA-Encoded

Solid-Phase Synthesis: Encoding Language Design and Complex Oligomer Library Synthesis. ACS Comb Sci 2015, 17, 518-534. 